\title{
DIFFERENTIAL EVOLUTION OPTIMIZATION FOR TARGETING SPACECRAFT MANEUVER PLANS
}

\section{Daniel Mattern}

NASA GSFC || Omitron, Inc.

2016 Astrodynamics Specialist Conference

13-16 September 2016

Long Beach, CA 


\section{BACKGROUND}

- Analysis performed for the Tracking and Data Relay Satellite (TDRS)

- Previous analysis examined the long-term impact to operational geosynchronous (GSO) region occupants if a TDRS at-risk spacecraft were to fail while on orbit

- Required at least $50 \mathrm{~km}$ "keep out zone" of GSO ring

- Found that "ideal" TDRS orbits had eccentricity of 0.004 and argument of perigee $(A \circ P)$ of $300^{\circ}$

- Only violation at perigee when AoP is aligned equatorially

- Lowest "allowable" eccentricity of 0.0012

- See references on last slide

- In 2015, TDRS-9 changed longitudes from $41^{\circ} \mathrm{W}$ to $12^{\circ} \mathrm{W}$

- This analysis examined if the "ideal" orbit parameters could be achieved with the drift termination (DT) maneuvers alone 


\section{PREVIOUS ANALYSIS}

\section{TARGET}

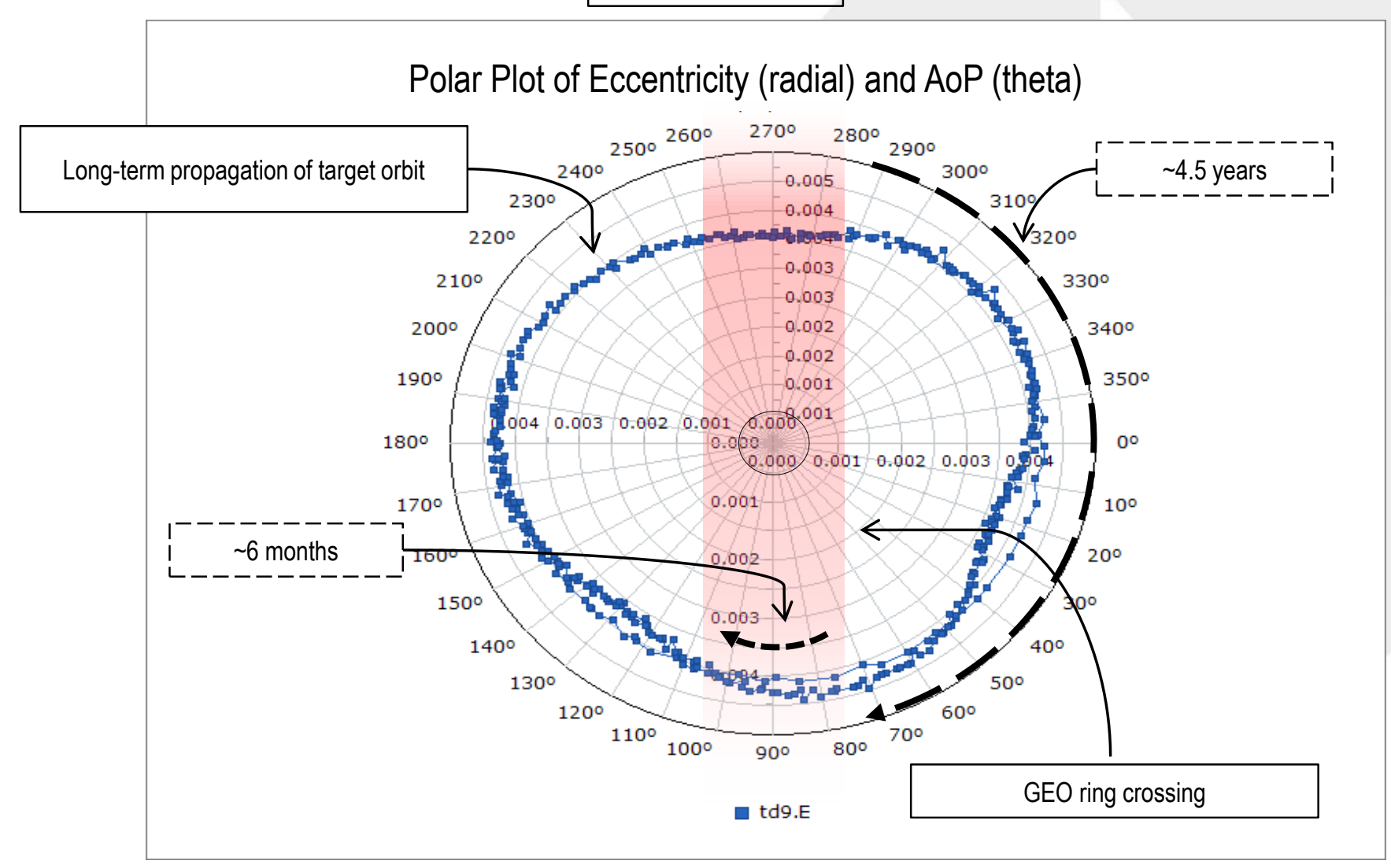




\section{CONSTRAINTS}

- Constraints

- Physical limits of the spacecraft thrusters based on tank and thruster temperatures

- All maneuver burn durations must be $\leq 10 \mathrm{sec}$

- Any 2 maneuvers must be $\geq 30$ minutes apart

- Final maneuver(s) must be on 10 June

- All maneuvers must be executed between 03:00 and 14:00Z on a given day 


\section{ASSUMPTIONS AND SIMPLIFICATIONS}

- Assumptions

- Only 18 DT maneuvers

- Each maneuver can be approximated by an impulsive maneuver

- Radial: $\quad-3.136(10-5) \mathrm{km} / \mathrm{s}$

- In-track: $5.716(10-5) \mathrm{km} / \mathrm{s}$

- Cross-track: $-5.242(10-5) \mathrm{km} / \mathrm{s}$

\section{Provided by White Sands Complex} (WSC)

- Simplifications

- Maneuvers executed on the half hour (03:00, 03:30, etc.)

- The first DT maneuver occurs no sooner than 1 June with maneuvers evenly distributed over the days leading to 10 June

- 9 maneuvers/day for 2 days, 6 maneuvers/day for 3 days, etc. 


\section{APPROACH}

- Single maneuver effects

- Looking at maneuvers executed across the window and on different days

- Examined 18 May, 2 June, 4 June, 6 June, 8 June, and 10 June

- Differential Evolution Optimization

- A target ephemeris created using the "ideal" orbit parameters

- Maneuver schedule is the control parameter

- 7-day summed difference between target and resultant position is the cost function

- 4 maneuver scenarios examined

1. DT-0 days: All maneuvers executed on 10 June

2. DT-1 days: 9 maneuvers per day occurring on 9 and 10 June

3. DT-2 days: 6 maneuvers per day occurring on 8,9 , and 10 June

4. DT-5 days: 3 maneuvers per day occurring on $5,6,7,8,9$, and 10 June 
Single MANEUVER EfFECTS 


\section{Single MANEUVER EFFECTS}

- Needed to qualitatively assess how different maneuver epochs would effect the target orbital parameters

- Maneuver epochs could be varied across days and across the daily maneuver window

- Specifically looking for

- Maneuver window effects

- Competing target constraints

- Maneuver day effects

- Possible changes in end-state proximity to target state 


\section{ONIAN END State Effects: MANEUVER ON 2 JUNE}

Shaping the Future of Aerospace

SMA Difference

24.570

0.0001

Eccentricity Difference

0.0001

$-0.618$

$-0.625$

80.6

4

\section{AoP Difference}

Longitude Difference 


\section{ONIA END State Effects: MANEUVER ON 4 JUNE}

Shaping the Future of Aerospace

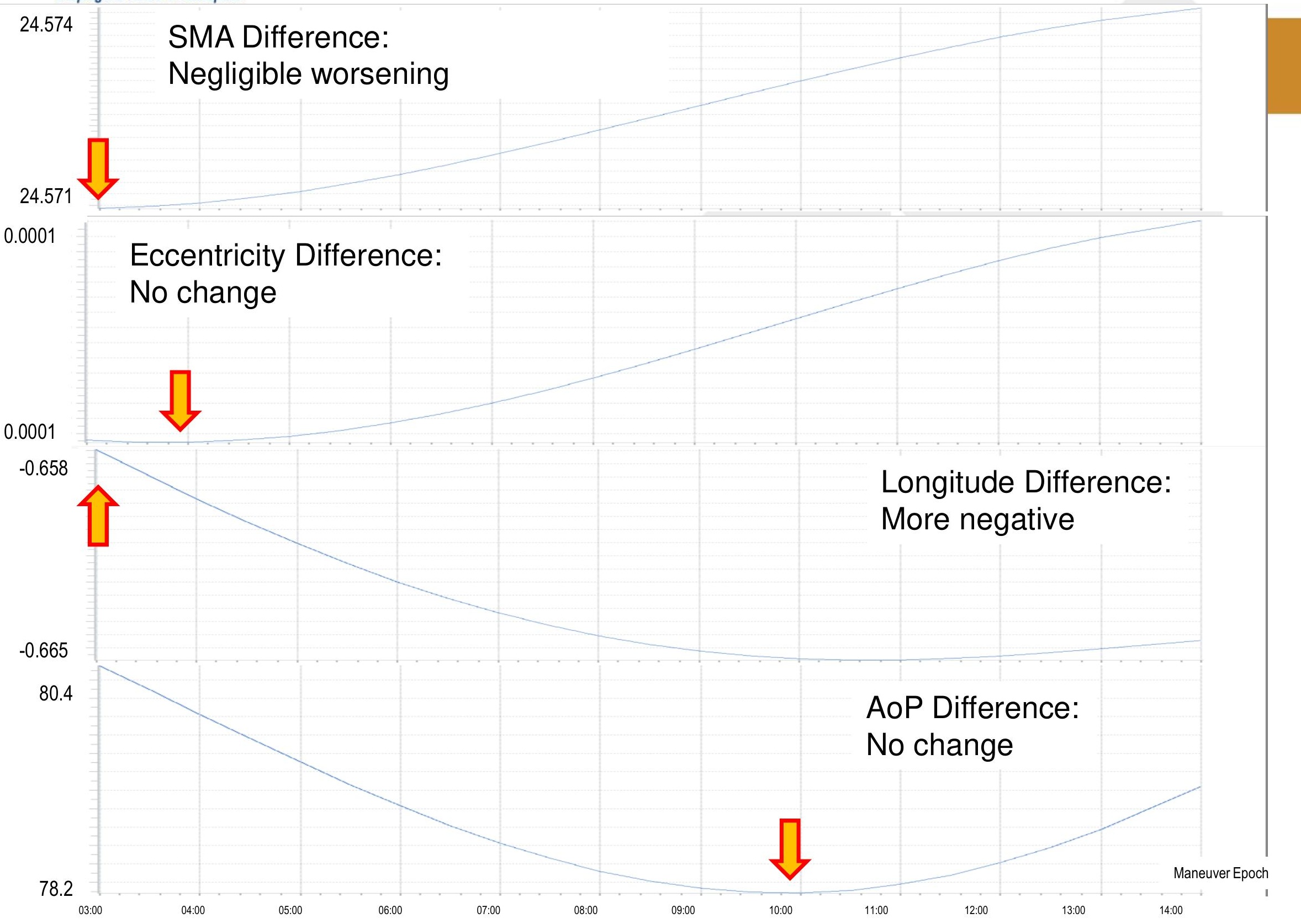




\section{ONIA END State Effects: MANEUVER ON 6 JUNE}

Shaping the Future of Aerospace

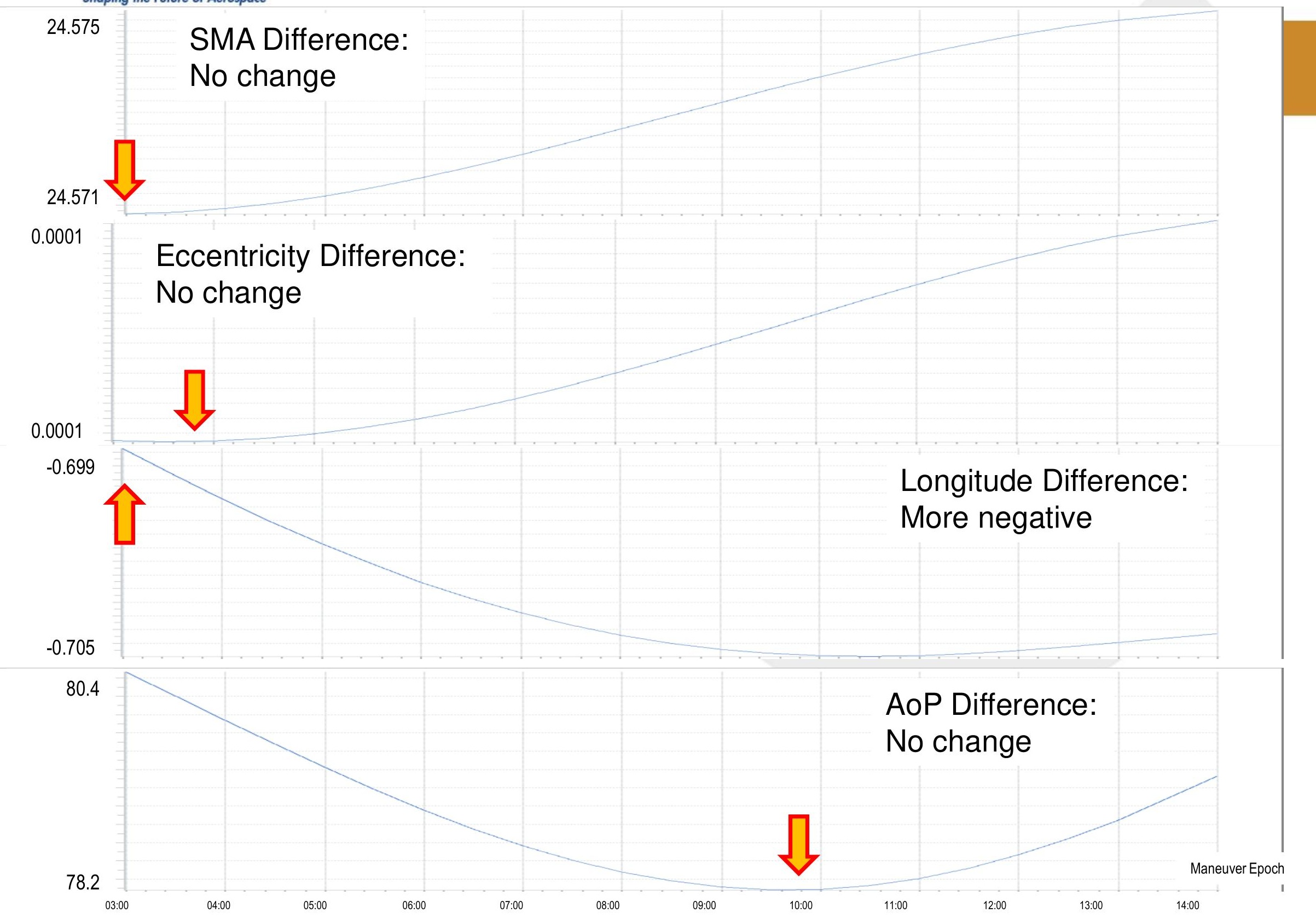




\section{ONIA END State Effects: MANEUVER ON 8 JUNE}

Shaping the Future of Aerospace

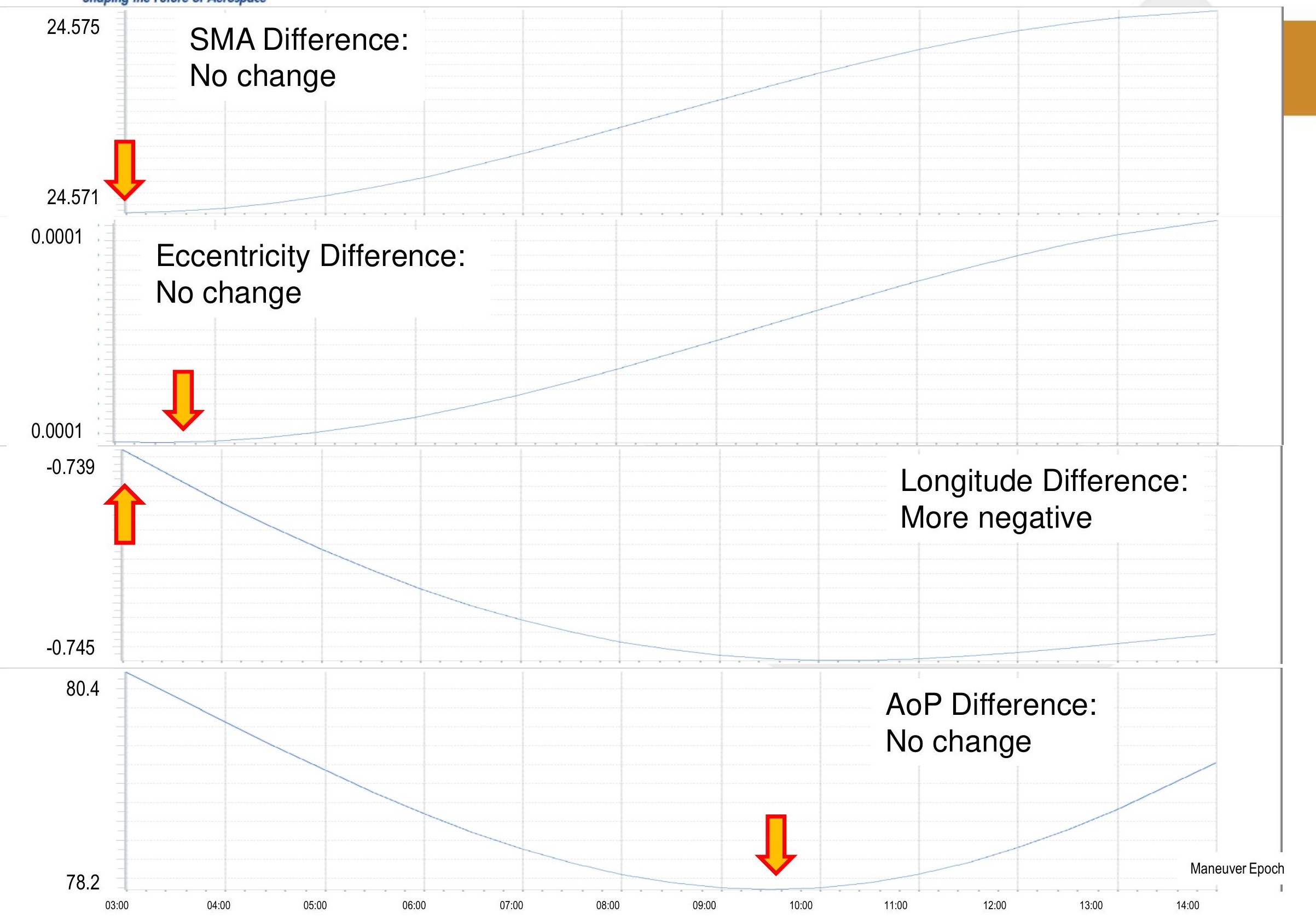




\section{OAINA END STATE EfFECTS: MANEUVER ON 10 JUNE}

Shaping the Future of Aerospace

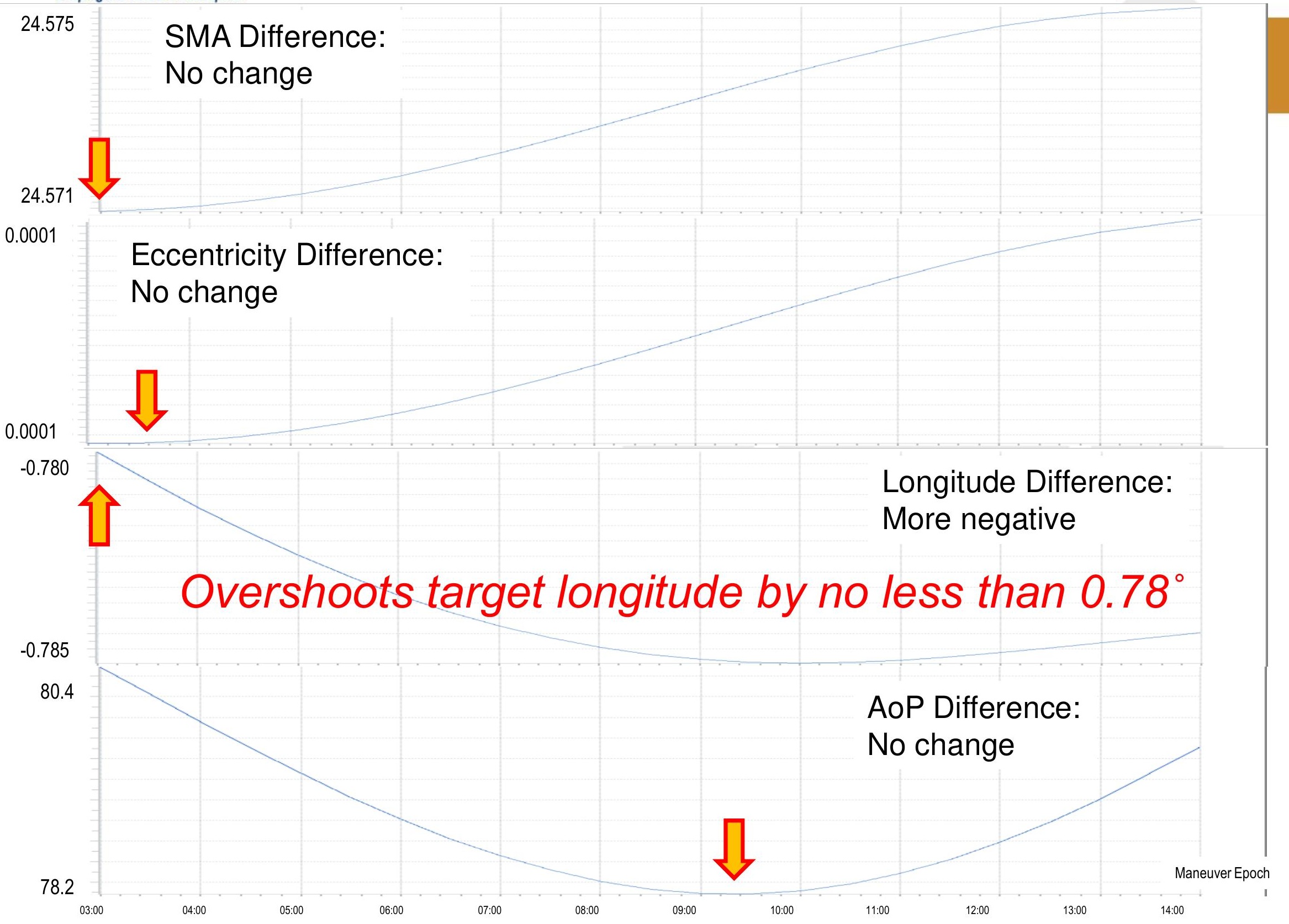




\section{SINGLE MANEUVER ANALYSIS}

- Maneuver Day Effects

- Waiting to maneuver closer to 10 June increases the likelihood of overshooting the target longitude.

- SMA, eccentricity, and AoP are marginally effected by changes in maneuver day

- Maneuver Window Effects

- There are competing constraints

- Maneuvering early in the window improves targeting SMA and eccentricity, but is bad for targeting AoP (and vice versa) 
Optimized MANEuVER ScEnARIOS 


\section{OPTIMIZED SCENARIOS}

- Starting with a target state, created a 7-day ephem

- Varied maneuver schedule to minimize target-toresultant ephem difference

- Assumptions, constraints, and simplifications resulted in $10^{10}$ possible maneuver schedule combinations

- Also looked at end-states which met the on-station longitude requirements

- Mean daily official edges: $12^{\circ} \mathrm{W} \pm 0.3^{\circ}$

- Daily East-most drift tolerance: $11.5^{\circ} \mathrm{W}$ (expected) 


\section{DT-0 DAYS}

Shaping the Future of Aerospace

Maneuver Window DT - 0 days (June 10)

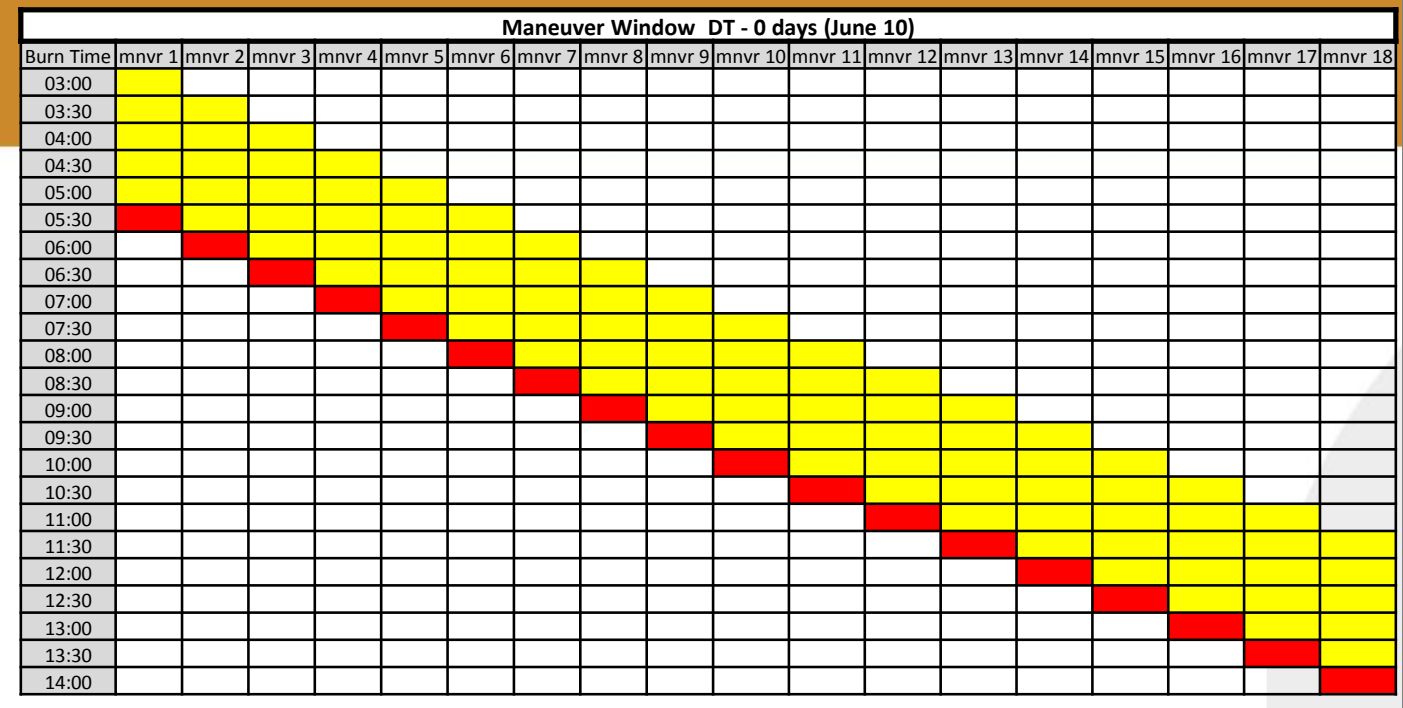

- Optimized maneuver plan

- 10 Jun 2015

- Every half hour from 05:30 to $14: 00$

- At 14:00Z on 10 June

- Longitude: $11.2^{\circ} \mathrm{W}$

- Eccentricity: 0.0011

- Arg. of Perigee: $248.6^{\circ}$

\section{TARGET}

Polar Plot of Eccentricity (radial) and AoP (theta)
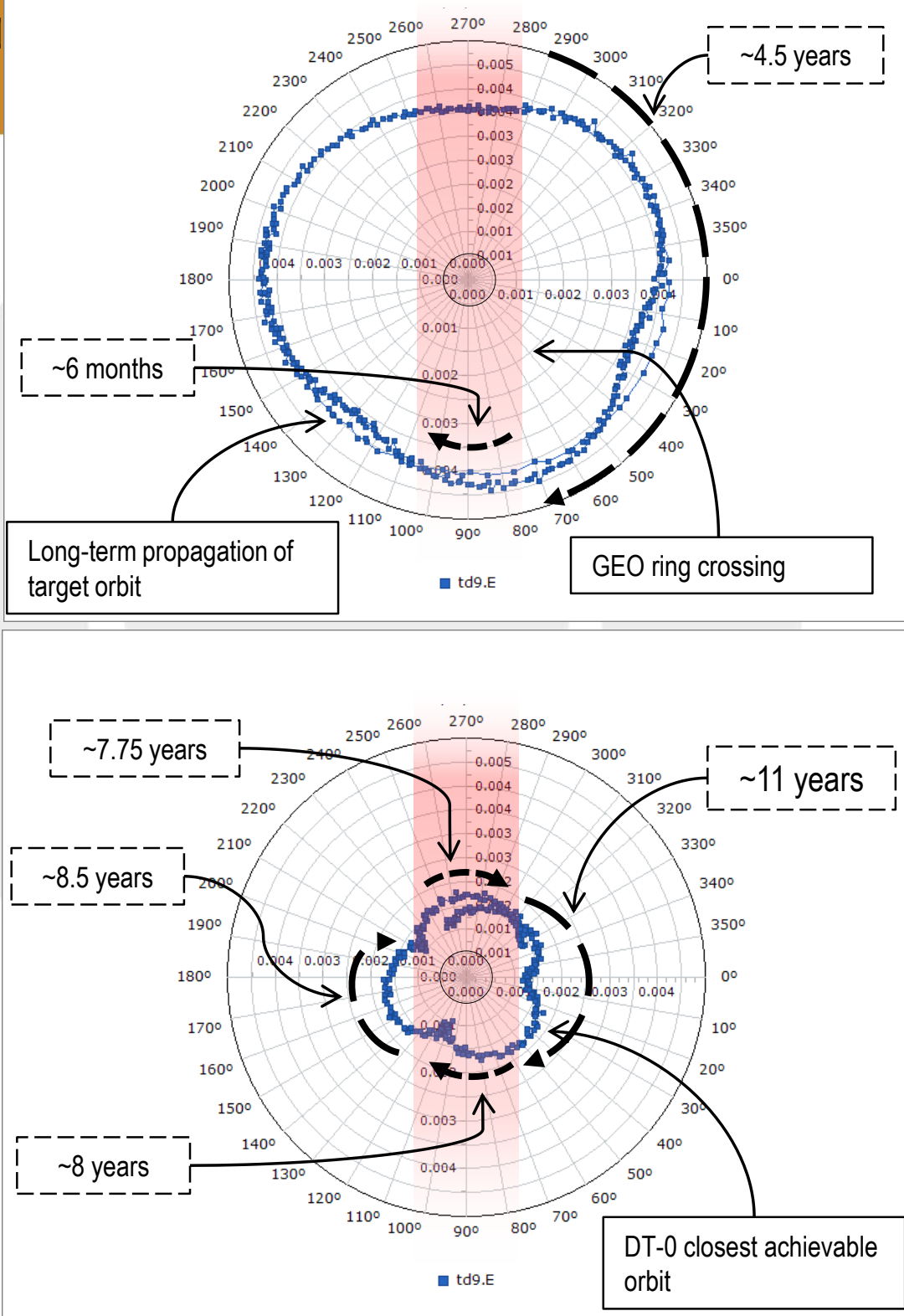


\section{DT-1 DAYS}

Shaping the Future of Aerospace

\begin{tabular}{|c|c|c|c|c|c|c|c|c|c|}
\hline \multicolumn{7}{|c|}{ Maneuver Window DT - 1 days (June 9, 10) } \\
\hline Burn Time & mnvr 1 & mnvr 2 & mnvr 3 & mnvr 4 & mnvr 5 & mnvr 6 & mnvr 7 & mnvr 8 & mnvr 9 \\
\hline $03: 00$ & & & & & & & & & \\
\hline $03: 30$ & & & & & & & & & \\
\hline $04: 00$ & & & & & & & & & \\
\hline $04: 30$ & & & & & & & & & \\
\hline $05: 00$ & & & & & & & & & \\
\hline $05: 30$ & & & & & & & & & \\
\hline $06: 00$ & & & & & & & & & \\
\hline $06: 30$ & & & & & & & & & \\
\hline $07: 00$ & & & & & & & & & \\
\hline $07: 30$ & & & & & & & & & \\
\hline $08: 00$ & & & & & & & & & \\
\hline $08: 30$ & & & & & & & & & \\
\hline $09: 00$ & & & & & & & & & \\
\hline $09: 30$ & & & & & & & & & \\
\hline $10: 00$ & & & & & & & & & \\
\hline $10: 30$ & & & & & & & & & \\
\hline $11: 00$ & & & & & & & & & \\
\hline $11: 30$ & & & & & & & & & \\
\hline $12: 00$ & & & & & & & & & \\
\hline $12: 30$ & & & & & & & & & \\
\hline $13: 00$ & & & & & & & & & \\
\hline $13: 30$ & & & & & & & & & \\
\hline $14: 00$ & & & & & & & & & \\
\hline
\end{tabular}

- Optimized maneuver plan

- 9, and 10 Jun 2015

- Every half hour from 10:00 to 14:00

- At $14: 00 Z$ on 10 June

- Longitude: $11.4^{\circ} \mathrm{W}$

- Eccentricity: 0.0008

- Arg. of Perigee: $258.0^{\circ}$

\section{TARGET}

Polar Plot of Eccentricity (radial) and AoP (theta)
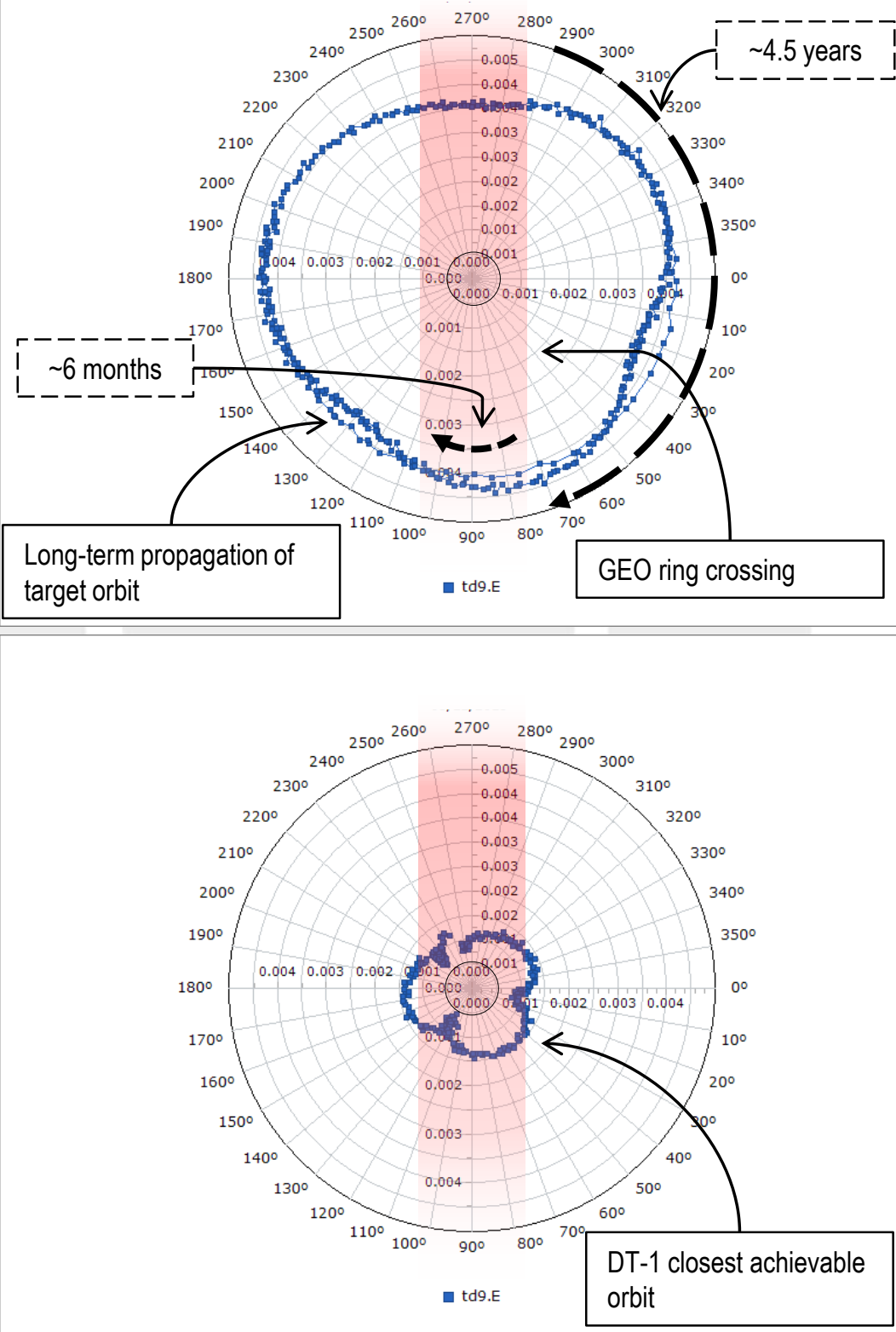


\section{DT-2 DAYS}

\section{TARGET}

\begin{tabular}{|c|l|l|l|l|l|l|l|}
\hline \multicolumn{6}{|c|}{ Maneuver Window DT - 2 days (June 8, 9, 10) } \\
\hline Burn Time & mnvr 1 & mnvr 2 & mnvr 3 & mnv 4 & mnvr 5 & mnvr 6 \\
\hline $03: 00$ & & & & & & \\
\hline $03: 30$ & & & & & & \\
\hline $04: 00$ & & & & & & \\
\hline $04: 30$ & & & & & & \\
\hline $05: 00$ & & & & & & \\
\hline $05: 30$ & & & & & & \\
\hline $06: 00$ & & & & & & \\
\hline $06: 30$ & & & & & & \\
\hline $07: 00$ & & & & & & \\
\hline $07: 30$ & & & & & & \\
\hline $08: 00$ & & & & & & \\
\hline $08: 30$ & & & & & & \\
\hline $09: 00$ & & & & & & \\
\hline $09: 30$ & & & & & & \\
\hline $10: 00$ & & & & & & \\
\hline $10: 30$ & & & & & & \\
\hline $11: 00$ & & & & & & \\
\hline $11: 30$ & & & & & & \\
\hline $12: 00$ & & & & & & \\
\hline $12: 30$ & & & & & & \\
\hline $13: 00$ & & & & & & \\
\hline $13: 30$ & & & & & & \\
\hline $14: 00$ & & & & & & \\
\hline
\end{tabular}

Polar Plot of Eccentricity (radial) and AoP (theta)

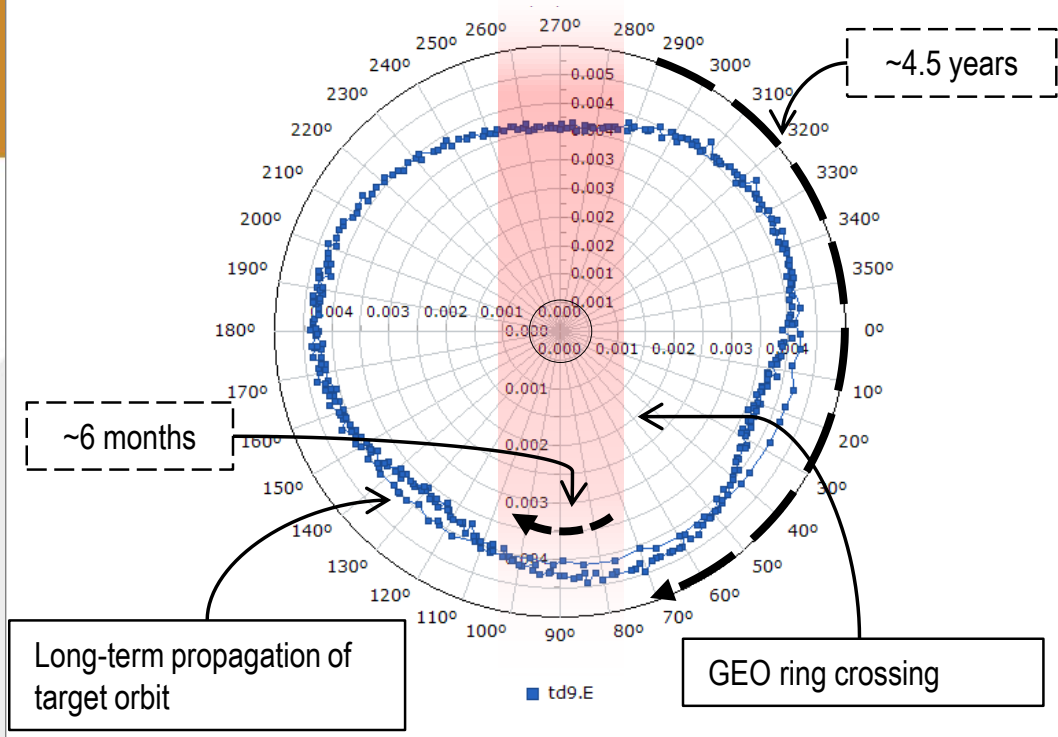

- Optimized maneuver plan

- 8,9 , and 10 Jun 2015

- Every half hour from 11:30 to 14:00

- At 14:00Z on 10 June

- Longitude: $11.6^{\circ} \mathrm{W}$

- Eccentricity: 0.0007

- Arg. of Perigee: $258.3^{\circ}$

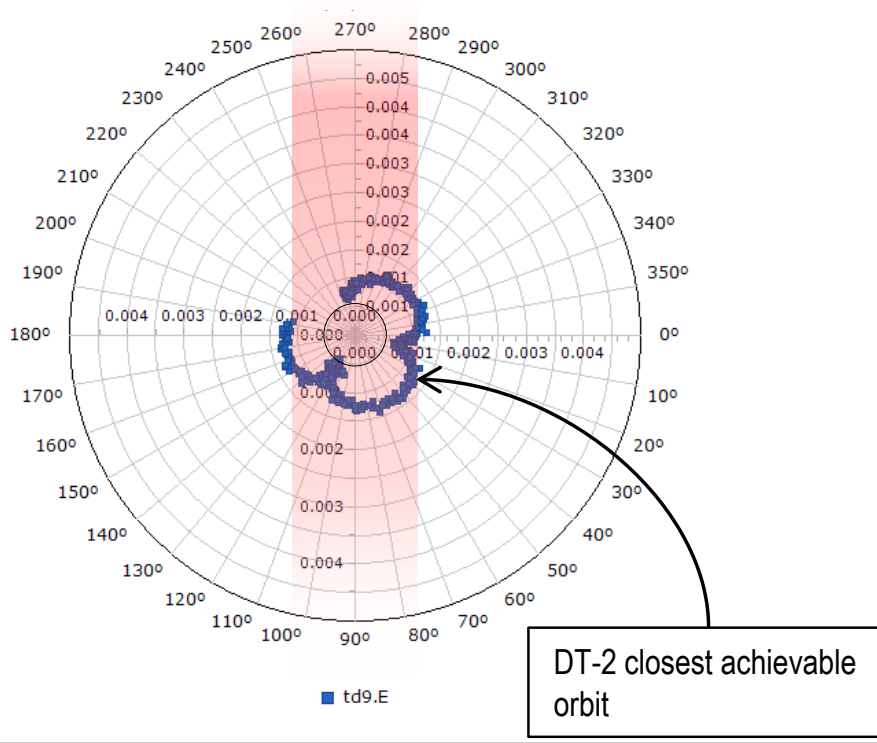




\section{DT-5 DAYS}

\section{TARGET}

\begin{tabular}{|c|c|c|c|}
\hline \multicolumn{4}{|c|}{ Maneuver Window DT - 5 days (June 5, 6, 7, 8, 9, 10) } \\
\hline Burn Time & mnvr 1 & mnvr 2 & mnvr 3 \\
\hline $03: 00$ & & & \\
\hline $03: 30$ & & & \\
\hline $04: 00$ & & & \\
\hline $04: 30$ & & & \\
\hline $05: 00$ & & & \\
\hline $05: 30$ & & & \\
\hline $06: 00$ & & & \\
\hline $06: 30$ & & & \\
\hline $07: 00$ & & & \\
\hline $07: 30$ & & & \\
\hline $08: 00$ & & & \\
\hline $08: 30$ & & & \\
\hline $09: 00$ & & & \\
\hline $09: 30$ & & & \\
\hline $10: 00$ & & & \\
\hline $10: 30$ & & & \\
\hline $11: 00$ & & & \\
\hline $11: 30$ & & & \\
\hline $12: 00$ & & & \\
\hline $12: 30$ & & & \\
\hline $13: 00$ & & & \\
\hline $13: 30$ & & & \\
\hline $14: 00$ & & & \\
\hline & & & \\
\hline
\end{tabular}

\section{Polar Plot of Eccentricity (radial) and AoP (theta)}

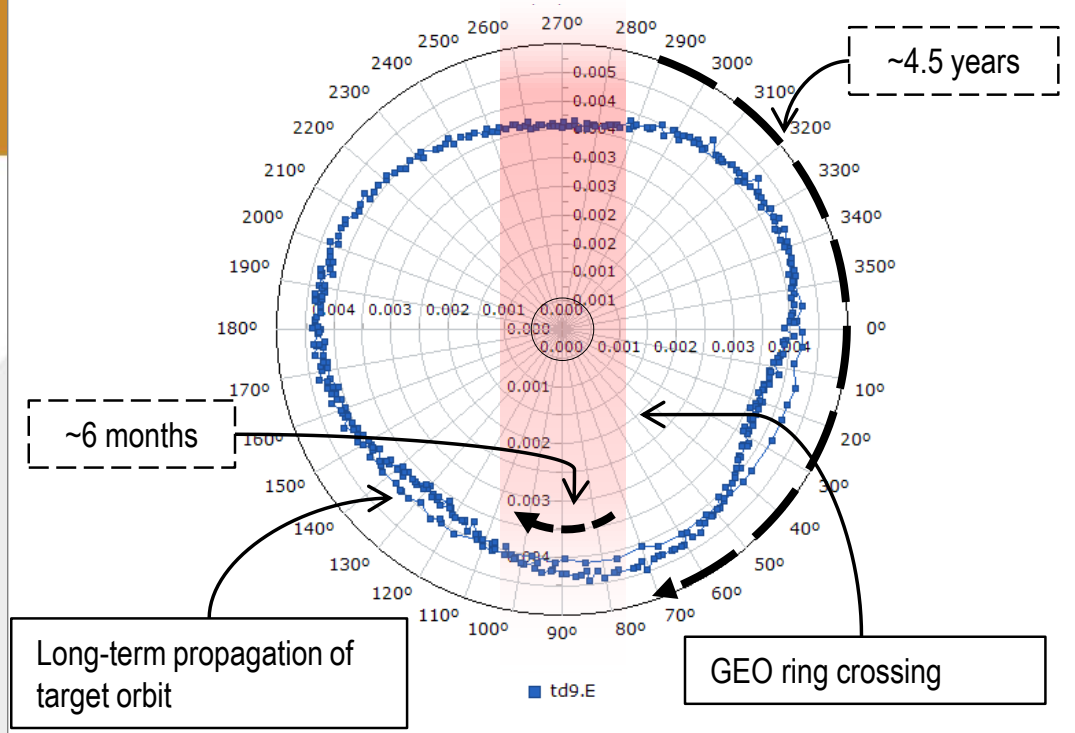

- Optimized maneuver plan

- 5, 6, 7, 8, 9, and 10 Jun 2015

- Every half hour from 13:00 to 14:00

- At 14:00Z on 10 June

- Longitude: $12.1^{\circ} \mathrm{W}$

- Eccentricity: 0.0006

- Arg. of Perigee: $256.1^{\circ}$

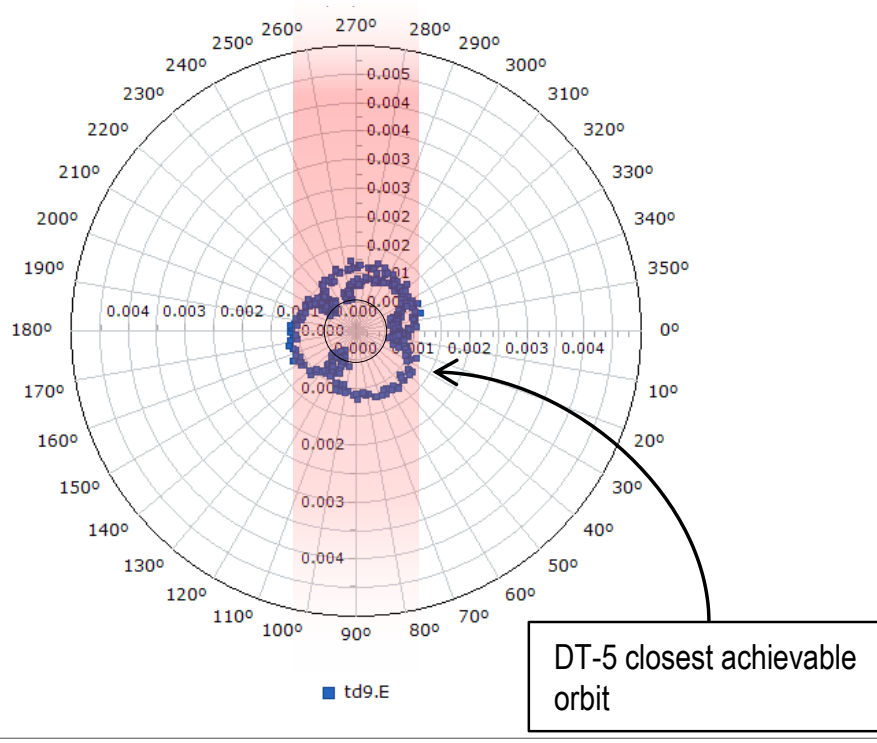




\section{RELAXED CONSTRAINTS}




\section{RELAXED CONSTRAINTS}

- Previous analyses suggested that the AoP constraint may be weighting the optimizer to target a greater change in AoP

- As a result, wanted to remove the AoP constraints to see if the optimizer would target a greater change in eccentricity

- Tested hypothesis using the DT-0 day, DT-2 day, and DT-5 day maneuver cadences

- Results

- Resultant optimizations targeted early window maneuver plans with better results

- Agreed with single-maneuver analysis 


\section{OAIAA \\ Shaping the Future of Aerospace}

\section{DT-0 EARLY WINDOW MANEUVERS}

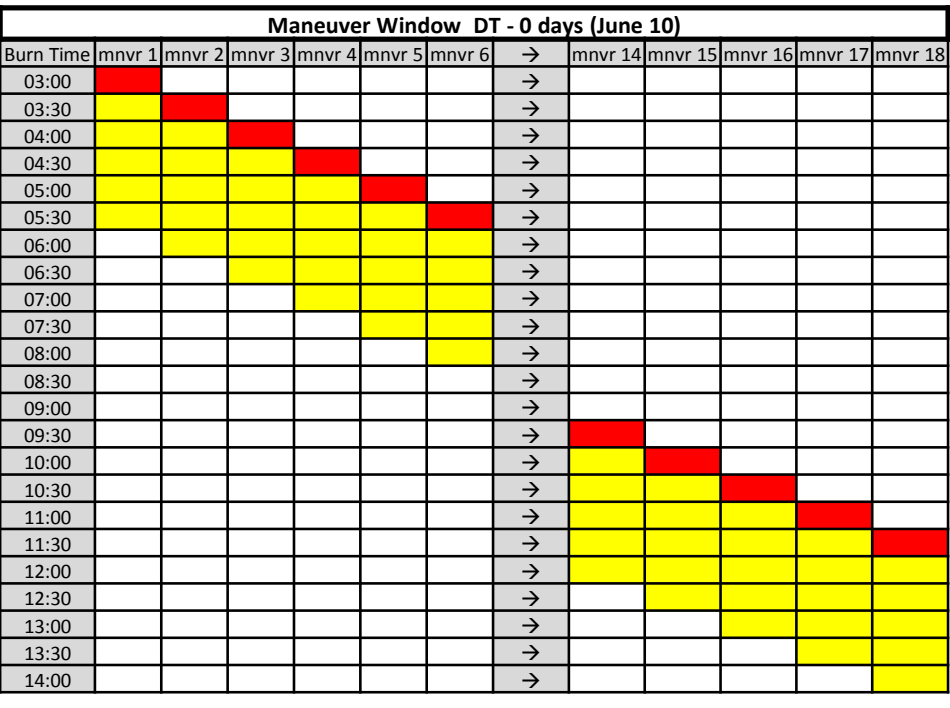

- At 14:00Z on 10 June

- Longitude: $11.2^{\circ} \mathrm{W}$

- Eccentricity: 0.0014

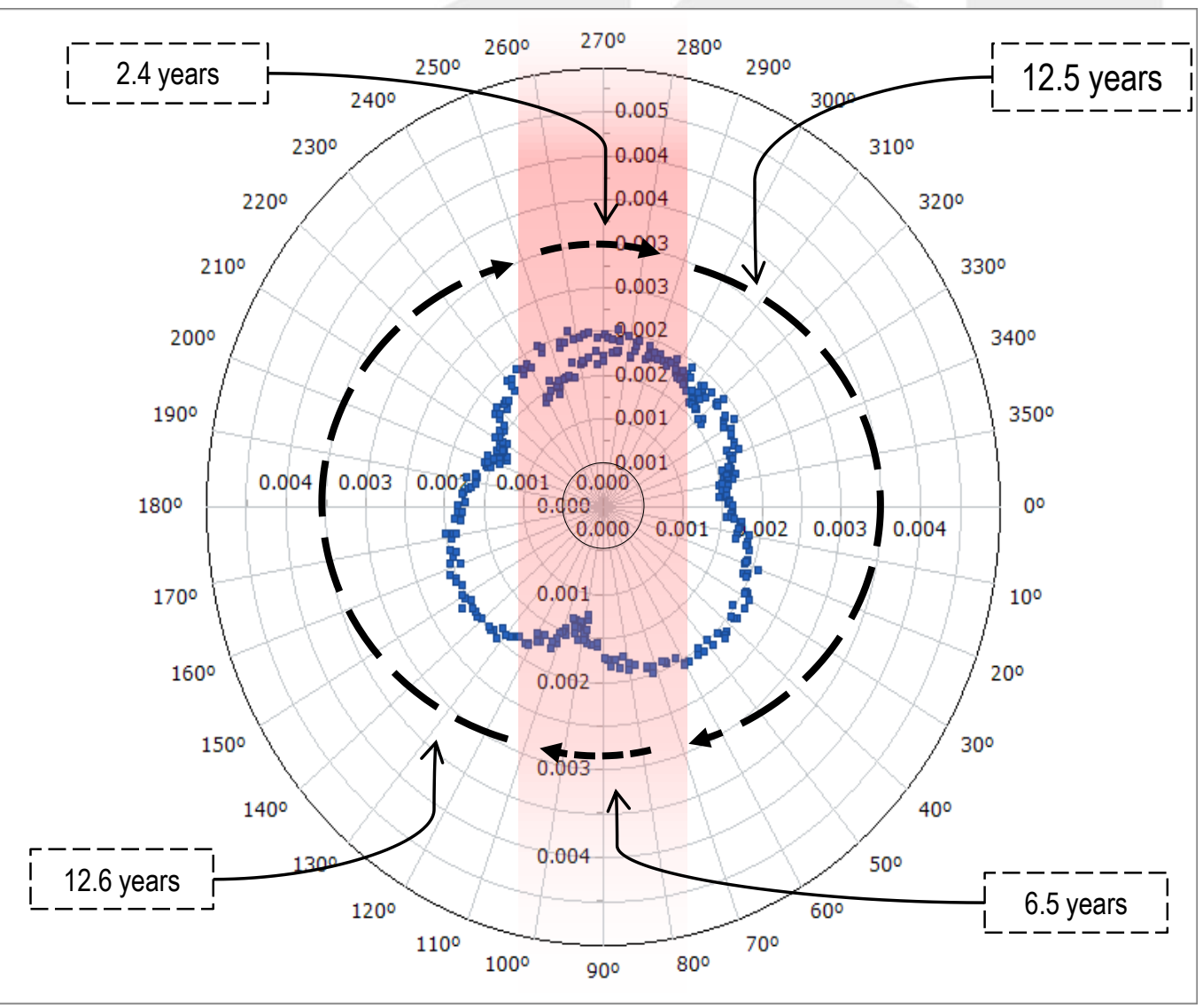




\section{OAIAA \\ Shaping the Future of Aerospace}

\section{DT-2 EARLY WINDOW MANEUVERS}

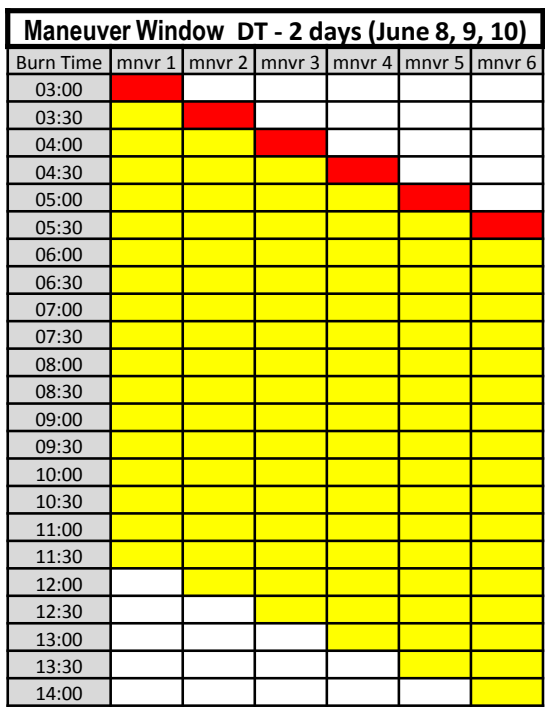

- At 14:00Z on 10 June

- Longitude: $11.65^{\circ} \mathrm{W}$

- Eccentricity: 0.0017

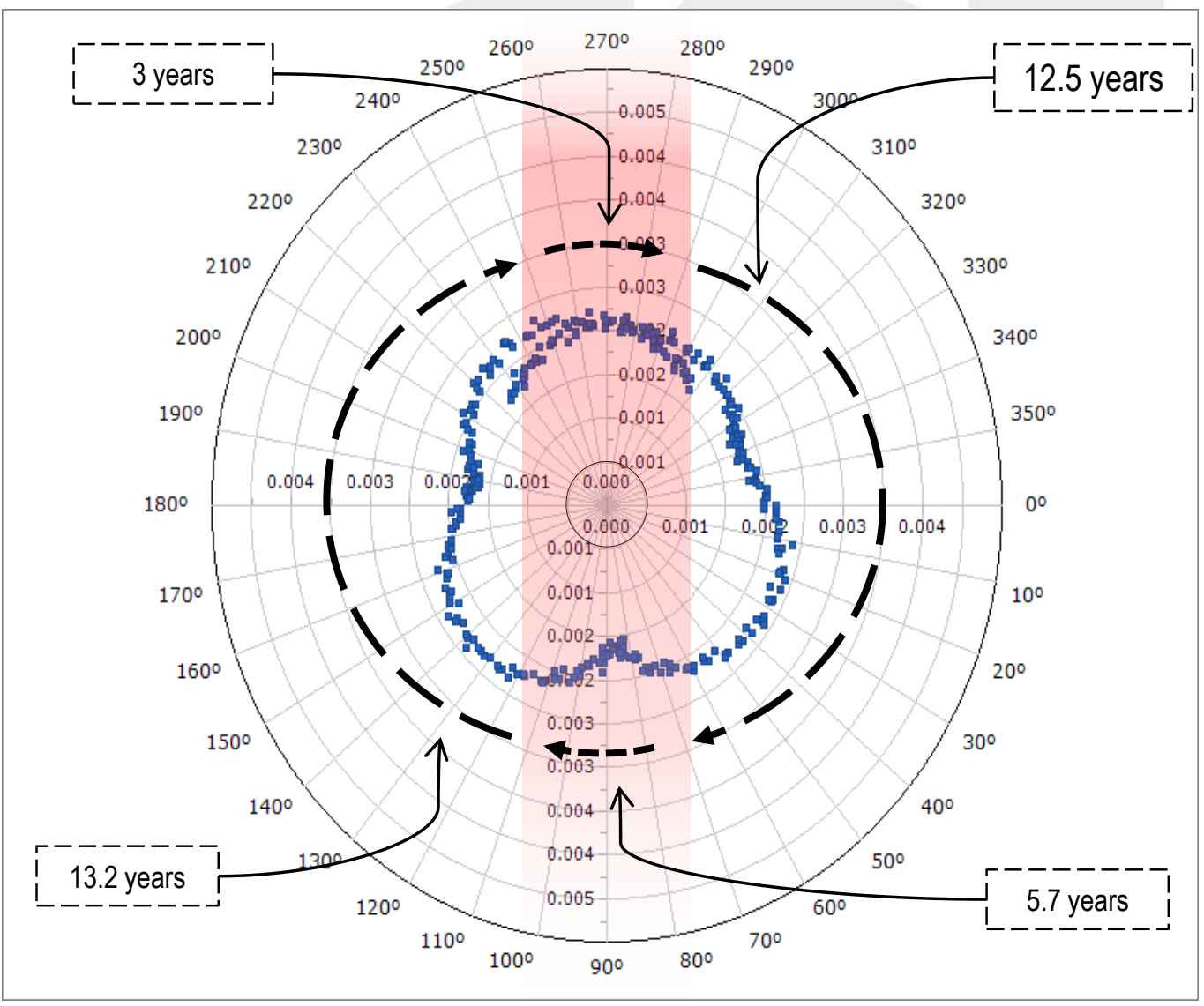




\section{OAIAA \\ Shaping the Future of Aerospace}

\section{DT-5 EARLY WINDOW MANEUVERS}

\begin{tabular}{|c|c|c|c|}
\hline \multicolumn{4}{|c|}{ Maneuver Window DT - 5 days (June $5,6,7,8,9,10$ ) } \\
\hline Burn Time & mnvr 1 & mnvr 2 & mnvr 3 \\
\hline \multirow{2}{*}{\multicolumn{4}{|c|}{$\frac{03: 00}{03: 30}$}} \\
\hline & & & \\
\hline \multicolumn{4}{|l|}{$04: 00$} \\
\hline \multicolumn{4}{|l|}{ 04:30 } \\
\hline \multicolumn{4}{|l|}{ 05:00 } \\
\hline \multirow{2}{*}{\multicolumn{4}{|c|}{ 05:30 }} \\
\hline & & & \\
\hline \multicolumn{4}{|l|}{$\frac{06: 00}{06: 30}$} \\
\hline \multicolumn{4}{|l|}{ 07:00 } \\
\hline \multicolumn{4}{|l|}{$07: 30$} \\
\hline \multicolumn{4}{|l|}{ 08:00 } \\
\hline \multicolumn{4}{|l|}{$08: 30$} \\
\hline \multirow{2}{*}{\multicolumn{4}{|c|}{$\begin{array}{l}09: 00 \\
09: 30\end{array}$}} \\
\hline \multirow{2}{*}{\multicolumn{4}{|c|}{$\frac{09: 30}{10: 00}$}} \\
\hline & & & \\
\hline \multicolumn{4}{|l|}{$10: 30$} \\
\hline \multicolumn{4}{|l|}{ 11:00 } \\
\hline \multicolumn{4}{|l|}{$11: 30$} \\
\hline \multirow{2}{*}{\multicolumn{4}{|c|}{$\begin{array}{l}\frac{12: 00}{12: 30} \\
\end{array}$}} \\
\hline \multirow{2}{*}{\multicolumn{4}{|c|}{$13: 00$}} \\
\hline & & & \\
\hline \multicolumn{4}{|l|}{ 13:30 } \\
\hline 14:00 & & & \\
\hline
\end{tabular}

- At 14:00Z on 10 June

- Longitude: $12.2^{\circ} \mathrm{W}$

- Eccentricity: 0.0017

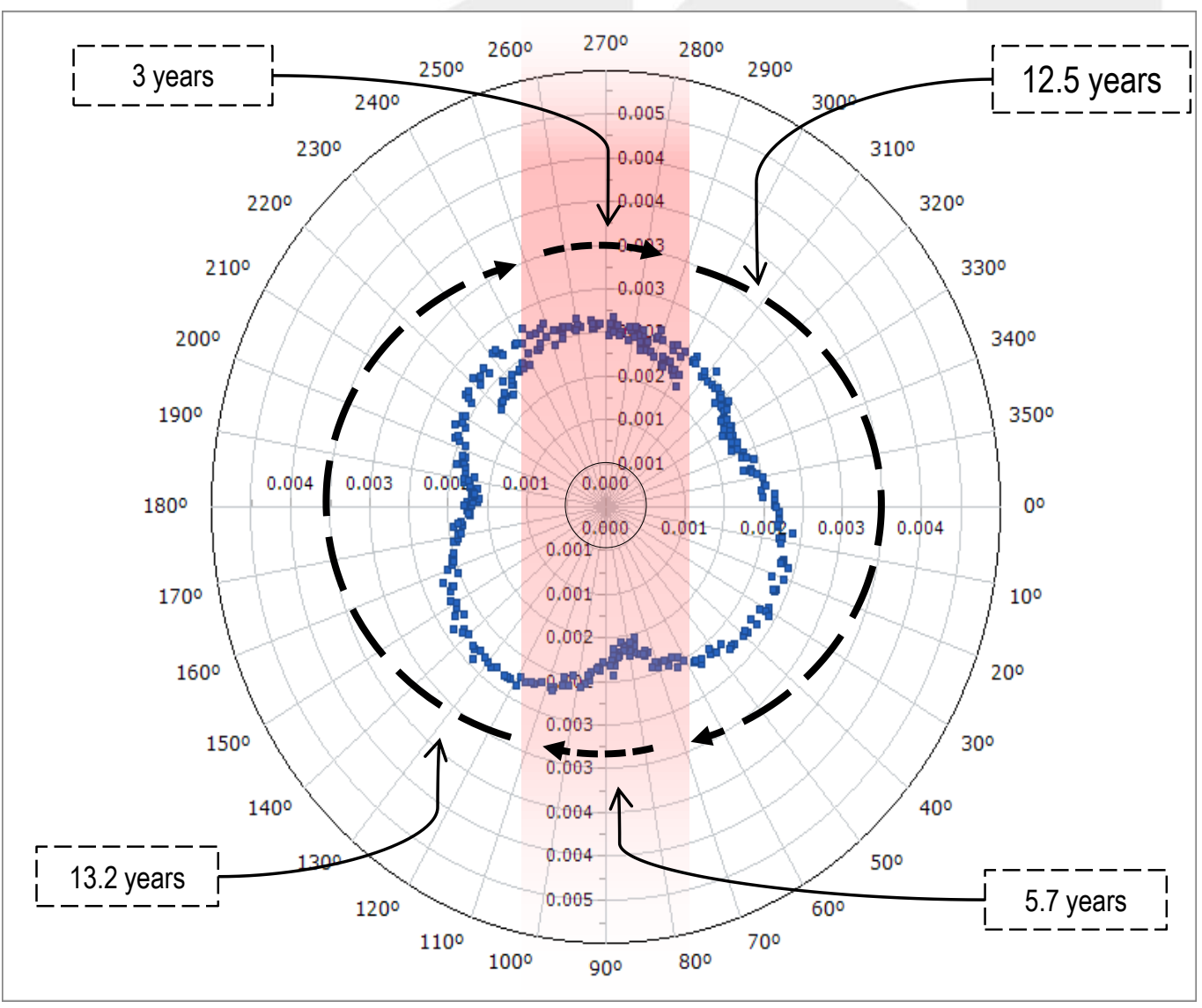




\section{CONCLUSIONS}

- To meet the original constraints $\left(\mathrm{AOP}=300^{\circ}, \mathrm{ecc}=0.004\right)$, the largest state change came from the AoP

- Optimizer originally targeted late window maneuver plans to accommodate

- By removing the AoP constraints, the optimizer began targeting maneuvers earlier in the window, thus producing larger changes in eccentricity

- AoP drifts continually clockwise, so this constraint is somewhat superfluous

- Optimization's best achieved eccentricity $\approx 0.0017$

- WSC eventual maneuver plan

- 10 DT maneuvers on 9 June

- 8 DT maneuvers and 1 small correction burn on 10 June

- All maneuver sequences began at the start of window and were executed every 30 minutes

- Resultant eccentricity $\approx 0.0015$ 


\section{REFERENCES}

- Dykes, A., Considering Orbit Changes for the Tracking and Data Relay Satellite System, NASA Orbital Debris Colloquium at GSFC, March 2002

- Cherney, R., et al., Eccentricity Management for TDRS Fleet, Presentation to Jon Walker (Code 452), 2005 


\section{Back-up Slides}




\section{OIIAN END StATE EfFECTS: MANEUVER ON 18 MAY}

Shaping the Future of Aerospace

SMA Difference

24.551

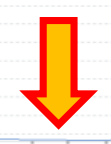

0.0001

Eccentricity Difference

0.0001

$-0.313$

$-0.324$

81.2

78.2
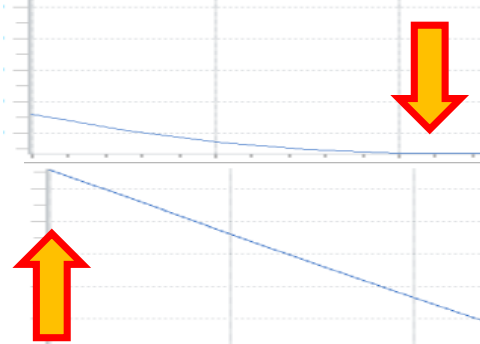

Longitude Difference

\section{AoP Difference}




\section{Additional Notes}

- Things to note:

- Waiting to execute maneuvers closer to or on 10 June caused the spacecraft to pass the target longitude (with current drift rate, should reach $12^{\circ} \mathrm{W}$ around 8 or 9 June)

- All maneuver plans resulted in an SMA at 14:00Z on 10 June of about $42166 \mathrm{~km}(2 \mathrm{~km}$ greater than GSO radius)

- Changes in SMA between "Early window" and "Late Window" maneuver plans were less than $1 \mathrm{~km}$

- Larger eccentricities will require larger East-most and West-most daily tolerances

- Recommendation delivered to WSC

- Reaching the target longitude on the target date needs to be the highest priority. Therefore, this analysis would suggest beginning the DT maneuvers before 10 June

- Executing burns earlier in the window should result in achieving a more desirable eccentricity

- Remaining $\Delta V$ provides a best achievable eccentricity of about 0.0017

- If desired, future station-keeping maneuvers may be used to further increase the eccentricity 


\section{OJIAa}

Shaping the Future of Aerospace 